\title{
Analytical Method of Process Capability for Steel
}

\author{
Wen-Pei Sung ${ }^{1}$, Kuen-Suan Chen ${ }^{2}$ and Cheer Germ Go ${ }^{3}$ \\ ${ }^{1}$ Department of Landscape Design and Management, ${ }^{2}$ Department of Industrial Engineering and Management, National Chin-Yi Institute \\ of Technology, Taichung, Taiwan; and ${ }^{3}$ Department of Civil Engineering, National Chung-Hsing University, Taichung, Taiwan
}

The mechanical properties of steel are the most important factor in the safety of structures that must be stable and appropriate. In order to investigate whether the process capability of steel production meets the quality requirements or not, Sung et al. proposed a procedure to evaluate the production and quality control of steel manufacturing. This evaluation model uses statistics to judge the quality of steel objectively. However, there are various types of steel which can satisfy the engineering requirements of design and construction, but procedures are lacking with which to evaluate the multi-process capability of the whole steelwork. In this paper, the objective evaluation methods proposed by Sung and Vännman are modified appropriately and extended by using the principles of statistical inference. A procedure to evaluate the quality of the multi-process capability analysis method is proposed. This evaluation method not only completely evaluates the process capability and quality, but it also improves the production process and quality control capability, and so can reduce production loss.

Keywords: Best estimators; Evaluation procedure; Fit index of steel; Stable index of steel; United confidence interval

\section{Introduction}

Taiwan and Japan are located around the Pacific seismic belt where earthquakes happen frequently. Thus, the capability of resisting earthquake-induced forces is the most important factor of a structure there. In engineering, steel is used to take tensile stress. The main reason is not only that the Poisson's ratio of steel approaches that of concrete, but that steels also provide good tensile strength and ductility. The investigation into the devastating Hanshin-Awaji and Chi-Chi earthquakes found that collapses of some of the structures were caused mainly by the tensile strength of the steel used being too high or too low.

Correspondence and offprint requests to: Dr W.-P. Sung, Department of Landscape Design and Management, National Chin-Yi Institute of Technology, Taiping, Taichung, 41111 Taiwan. E-mail: sung809@ chinyi.ncit.edu.tw
A steel structure is more reliable than a wooden structure or a reinforced concrete structure for resisting earthquake-induced forces $[1,2]$. According to these results, steel will be widely used in the construction industry in Taiwan and Japan. Carbon steel, high strength-low alloy steel and heat treatment-low alloy steel are often used as constructional materials for frame structures. Additionally, in order to promote the earthquake resistance capability of a structure, new steels for construction have been developed such as: low yielding ratio steel; narrow yielding stress variation steel; and low yielding stress steel.

To increase the strength and provide special functional characteristics to steel, alloying elements other than carbon are added to produce steels of various yielding stress. Quenching and tempering heat treatment procedures can also be used to obtain higher yielding steel. The key aspects of the production process are puddling and heat treatment after crushing. These techniques affect the mechanical properties of the steel. In order to ensure the quality of the steel, the analyses of chemical composition and mechanical behaviour are tested. The test of steel monotonically loaded in tension is widely used to ascertain the quality of steel that has a major effect on the safety of structures. Although there are some analytical methods for evaluating the production process control of steel, there is still a lack of evaluation methods to estimate the efficiency of the production processes control involved. The purpose of this work is to propose a procedure to evaluate the quality and control capability of the multi-processes of steelworks.

At this time, many new evaluation methods are continually being developed to enhance the analysis and the evaluation of a satisfactory production process, for the loss function of the production process and for the index of production capability. The technology of engineering quality analysis is very mature for assessing the production capability. Effective methods for evaluating production processes are provided by many workers such as Kane [3], Chan et al. [4], Chou and Owen [5], Boyles [6], Pearn et al. [7], Boyles [8], Greenwich and Jahr-Schaffrath [9], and Chen [10]. Sung et al. [11] proposed indices of accuracy and precision to evaluate the quality and tensile strength of steel bars. A new index, the quality index, for the tensile strength of steel reinforcing bars is created to evaluate the production quality and efficiency of steel bar manufacture by combining these two indices. This method is useful for 
improving and promoting the structural quality in the initial stage. $X$ represents the tensile strength of a steel reinforcing bar that is manufactured by one of the steelworks. The values of $X$ are not likely to be the same. Thus, Sung et al. [11] pointed out that $X$ is a random variable. Since the quality of structures is affected by the tensile strength of steel, the difference between actual tensile strength $X$ and target value $T$ should be less than $d$, which is called the tolerance error value. Consequently, the tensile strength value of steel, which is called the target value $T$, should be appropriate. The actual tensile strength of steel should result in a tolerance interval $T$ $\pm d$ in which the upper tolerance limit $U T L$ is from $T$ plus $d$ $(U T L=T+d)$ and the lowest tolerance limit $L T L$ is from $T$ minus $d(L T L=T-d)$. When the tensile strength of steel exceeds the upper tolerance limit or is below the lowest tolerance limit, the quality of steel does not meet the required specification. According to the above-mentioned procedure, the evaluation formulae for the index of fit and stability can be defined as follows:

$$
\begin{aligned}
& Q_{i f}=\frac{\mu-T}{d} \\
& Q_{i s}=\frac{\sigma}{d}
\end{aligned}
$$

where $\mu$ is the average tensile strength, and $\sigma^{2}$ is the variance value for the tensile strength of steel.

When $\mu$ approaches $T$, the fit degree reaches the standard. Therefore, in accordance with the definition of the index $Q_{i f}$, $Q_{i f}>0(\mu>T)$, it shows that the average tensile strength of the steel bars is greater than the target value $T$. On the other hand, $Q_{i f}<0(\mu<T)$ indicates that the average tensile strength of the steel bars is smaller than the target value $T$. Thus, the steelworks can improve the manufacturing procedure based on the index of fitness. The values of variance $\left(\sigma^{2}\right)$ represent the stability of the tensile strength of the steel. A lower value of $\sigma^{2}$ indicates a more stable tensile strength.

In this paper, the index proposed by Boyles [8] is used to evaluate the manufacturing process capability on the strength using the above indices. The relationship of the indices and the rate of resulting tolerances are investigated simultaneously. Finally, the procedure to evaluate the quality and the quality control capability of multi-process of steelworks is proposed by making use of the theoretical analytical principles of statistical inference and the evaluation proposed by Vännman and Deleryd [12]. This new procedure is not only useful for customers to examine and compare purchase decisions, but it is also the best way to improve the process capability of steelworks.

\section{The Quality Index of Steel and the Rate of Tallied Tolerance}

The indices of fit and stability of tensile strength of the steel are used to evaluate whether the fitness and stability meet the required specifications or not. The denominator $d$ of the index of stability is called the tolerance error value which can be specified in structural design criteria or in a contract or agreement between a purchasing unit and a manufacturer. When the process capability is stable and the assumed $d$ is a constant value, then it reveals that the variance value $\sigma$ of the tensile strength is small. This indicates that the index $Q_{i s}$ is small, so the quality of the steel is stable. Therefore, a smaller value of $Q_{i s}$ represents higher stability. The differences of the actual tensile strength of steel $X$, and target value $T$, are usually less than the tolerance error value $d$. This indicates that the tensile strength of steel attains the required specification, otherwise, the quality of the steel reinforcing bars is not acceptable. The rate of tallied tolerance $p$ can be calculated as $p=P(L T L \leq$ $X \leq U T L)$. The larger the value of $p$ the better is the quality of the steel. The rate of tallied tolerance $p$ can be defined by the following expression based on the assumption of normal distribution.

$$
\begin{aligned}
p & =P(L T L \leq X \leq U T L) \\
& =P\left(\frac{L T L-\mu}{\sigma} \leq Z \leq \frac{U T L-\mu}{\sigma}\right) \\
& =\left[\Phi\left(\frac{U T L-\mu}{\sigma}\right)-\Phi\left(\frac{L T L-\mu}{\sigma}\right)\right] \\
& =\Phi\left(\frac{U T L-\mu}{\sigma}\right)+\Phi\left(\frac{\mu-L T L}{\sigma}\right)-1
\end{aligned}
$$

where $P(\mathbf{)})$ is the cumulative probability density function of random variable $X$.

Owing to

$$
\begin{aligned}
\frac{U T L-\mu}{\sigma} & =\frac{(T+d)-\mu}{\sigma}=\left(\frac{d-(\mu-T)}{d}\right) \times\left(\frac{d}{\sigma}\right) \\
& =\left(1-\frac{\mu-T}{d}\right) /\left(\frac{\sigma}{d}\right) \\
& =\left(1-Q_{i f}\right) / Q_{i s}
\end{aligned}
$$

and

$$
\begin{aligned}
\frac{u-L T L}{\sigma} & =\frac{\mu-(T-d)}{\sigma}=\left(\frac{d+(\mu-T)}{d}\right) \times\left(\frac{d}{\sigma}\right) \\
& =\left(1+\frac{\mu-T}{d}\right) /\left(\frac{\sigma}{d}\right) \\
& =\left(1+Q_{i f}\right) / Q_{i s}
\end{aligned}
$$

where $U T L$ is the upper tolerance limit, and $L T L$ is the lowest tolerance limit.

The rate of tallied tolerance can be defined as follows:

$$
p=\left(\Phi\left\{\left(1-Q_{i f}\right) / Q_{i s}\right\}+\Phi\left\{\left(1+Q_{i f}\right) / Q_{i s}\right\}\right)-1,
$$

where $\Phi(\bullet)$ is the cumulative function of standard normal distribution.

When the value of $Q_{i f}$ is equal to " 0 " $(\mu=T)$, the index $Q_{i s}$ is the function of the rate of tallied tolerance $p$ as follows:

$$
p=2 \Phi\left(1 / Q_{i s}\right)-1
$$

In accordance with Eq. (4), when the value of $Q_{i s}$ is low, it indicates that the rate of tallied tolerance is high. For example, when the values of the index $Q_{i s}$ are $\frac{1}{3}, \frac{1}{2}$, and 1 , then the rate of tallied tolerance $p$ can be calculated by using Eq. (4), as shown as follows: 


$$
\begin{aligned}
& Q_{i s}=\frac{1}{3} \Rightarrow p=2 \Phi(1 /(1 / 3))-1=2 \Phi(3)-1=99.73 \% \\
& Q_{i s}=\frac{1}{2} \Rightarrow p=2 \Phi(1 /(1 / 2))-1=2 \Phi(2)-1=95.44 \% \\
& Q_{i s}=1 \Rightarrow p=2 \Phi(1 / 1)-1=2 \Phi(1)-1=68.27 \%
\end{aligned}
$$

Thus, the rate of tallied tolerance $p$ is $99.73 \%, 95.44 \%$, and $68.27 \%$. A lower value of $Q_{i s}$ indicates a more stable tensile strength of the steel and a higher rate of tallied tolerance $p$. Thus, the index of stability satisfactorily reflects the rate of tallied tolerance. Table 1 is the relationship of the rate of tallied tolerance between $Q_{i s}$ and $Q_{i f}$.

If the value of $Q_{i s}$ is calculated, then the rate of tallied tolerance $p$ can be easily calculated from Table 1 . In conclusion, Eq. (3) can not only evaluate the indices of fit and stability of the tensile strength of steel easily, but can also easily evaluate the rate of tallied tolerance for the steelworks.

The index to evaluate the production process capability proposed by Boyles [8] is used to evaluate the process capability of the steel. The definition of this index is shown as follows:

$$
S_{p k}=\frac{1}{3} \Phi^{-1}\left\{\frac{1}{2} \Phi\left(\frac{U T L-\mu}{\sigma}\right)+\frac{1}{2} \Phi\left(\frac{\mu-L T L}{\sigma}\right)\right\}
$$

Because $(U T L-\mu) / \sigma=\left(1-Q_{i f}\right) / Q_{i s}$ and $(\mu-L T L) / \sigma=$ $\left(1+Q_{i f}\right) / Q_{i s}$, the index $S_{p k}$ can be redefined as follows:

$$
\begin{aligned}
& S_{p k}=\frac{1}{3} \Phi^{-1}\left\{\frac{1}{2} \Phi\left[\left(1-Q_{i f}\right) / Q_{i s}\right]\right. \\
& \left.+\frac{1}{2} \Phi\left[\left(1+Q_{i f}\right) / Q_{i s}\right]\right\}
\end{aligned}
$$

According to Eqs (3) and (5), the relationship between the index $S_{p k}$ and the rate of tallied tolerance $p$ of steel can be shown as follows:

$$
p=2 \Phi\left(3 S_{p k}\right)-1
$$

When the value of index $S_{p k}$ is large, the rate of tallied tolerance is high. For example, when the index $S_{p k}=1.0$, it ensures that the tensile strength of the steel meets the rate of tallied tolerance $p=99.73 \%$. The relationship between $S_{p k}$ and $p$ is shown in Table 2.

\section{Multi-Process Capability Analysis Chart of Steel}

To satisfy the different requirements of manufacturers and construction engineers, various tensile strengths and types of steel are produced. Because of the different production processes or different types of steel, the target values and tolerance limits vary. Even though the evaluation model proposed by Sung et al. [11] is a useful method to judge the quality of
Table 2. Value of $S_{p k}$ and the corresponding values of $p$.

\begin{tabular}{ll}
\hline$S_{p k}$ & $\begin{array}{l}\text { The rate of tallied } \\
\text { tolerance } p\end{array}$ \\
\hline$\frac{1}{3}$ & 0.628689492 \\
$\frac{2}{3}$ & 0.954499736 \\
1 & 0.997300231 \\
$\frac{4}{3}$ & 0.999936658 \\
$\frac{5}{3}$ & 0.999999426 \\
2 & 0.999999998 \\
\hline
\end{tabular}

steel objectively, nevertheless, this method cannot be used to evaluate different production processes simultaneously. Therefore, a set of procedures to judge the production and quality control capability of multi-process steelworking is proposed in this paper. This is a complete evaluation method for multiprocess capability. In order to set up this evaluation model, the indices proposed by Boyles [8], Sung et al. [11] and the evaluation method proposed by Vännman and Deleryd [12] are used and modified. The principles of statistical inference and theoretical analysis are then used to establish a set of procedures to evaluate the quality of the multi-process capability of the steel, the quality of the production process and the quality control. According to the different production processes for various types of steel, the specification for the various types of steel also varies. Hence, $Q_{i f}$ is used to replace $\mu$ as the abscissa in the graph, and $Q_{i s}$ is used to replace $\sigma$ as the ordinate. Then, the contour of $S_{p k}$ can be plotted as in Fig. 1 which is called a multi-process capability analysis chart. Thus, the different specifications of the production processes of steel can be used to plot the coordinate points of $\left(Q_{i}, Q_{i s}\right)$ for different production processes on the same analysis chart. The four contour lines from top to bottom shown in Fig. 1 represent the corresponding values of $S_{p k}$ which are 1.00, 1.33, 1.50, and 2.00, respectively. Pearn and Chen [13] pointed out that the requirements of the production processes can be classified into five conditions (Table 3).

The coordinate points of $\left(Q_{i}, Q_{i s}\right)$ for various type of steel in the multi-process capability analysis chart can be used to analyse the production capability of the process by a quality control engineer. When the coordinate point of the production process is located in block I, it reveals that the production capability of the process is inadequate. An improvement in the production process can be decided upon based on positive or

Table 1. The relationship of $p$ between $Q_{i s}$ and $Q_{i f}$.

\begin{tabular}{lllllll}
\hline$Q_{i s}$ & $Q_{i f}= \pm 0.0$ & $Q_{i f}= \pm 0.2$ & $Q_{i f}= \pm 0.4$ & $Q_{i f}= \pm 0.6$ & $Q_{i f}= \pm 0.8$ & $Q_{i f}= \pm 1.0$ \\
\hline 1 & 0.31731 & 0.32693 & 0.35501 & 0.39938 & 0.45667 & 0.50000 \\
2 & 0.04550 & 0.06300 & 0.11762 & 0.21254 & 0.34474 & 0.50000 \\
3 & 0.00270 & 0.00836 & 0.03594 & 0.11507 & 0.27425 & 0.50000 \\
4 & 0.00006 & 0.00069 & 0.00820 & 0.05480 & 0.21186 & 0.50000 \\
\hline
\end{tabular}




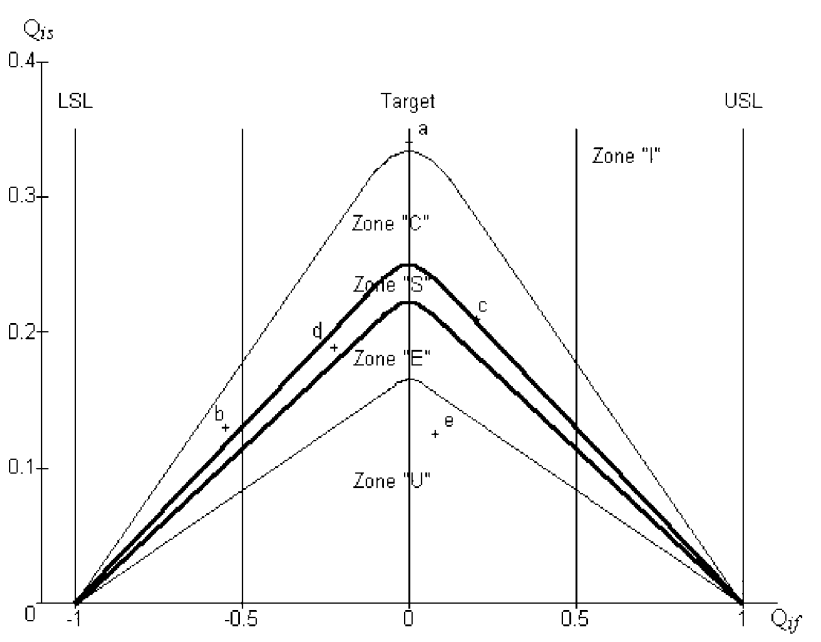

Fig. 1. Multi-process capability analysis chart for five production processes.

Table 3. The index $S_{p k}$ and correspondence quality situation.

\begin{tabular}{lll}
\hline Capability indices & Situation of quality & $\begin{array}{l}\text { Region of } \\
\text { production } \\
\text { process capability }\end{array}$ \\
\hline$-\infty<S_{p k}<1.00$ & Inadequate & $\mathrm{I}$ \\
$1.00 \leq S_{p k}<1.33$ & Capability & $\mathrm{C}$ \\
$1.33 \leq S_{p k}<1.50$ & Satisfactory & $\mathrm{S}$ \\
$1.50 \leq S_{p k}<2.00$ & Excellent & $\mathrm{E}$ \\
$2.00 \leq S_{p k}<\infty$ & Super & $\mathrm{U}$ \\
\hline
\end{tabular}

negative values of $Q_{i f}$ and the magnitude of $Q_{i s}$. Therefore, a value of the index $S_{p k}$ can be obtained from multi-process capability analysis. The rate of tallied tolerance of tensile strength of the steel can also be acquired easily based on the one-to-one mathematical relationship of index $S_{p k}$ and the rate of tallied tolerance $p$ of the steel. Moreover, the deviation and variation degree of this production process can be evaluated quickly. Thus the required improvement for an inadequate production process can be obtained objectively.

\section{Analytical Method for Multi-Process Capability of Steel}

Chang et al. [14] pointed out that the parameters of the production process are unknown. Therefore, an estimated value of the index must be obtained by means of a sample. Because of sampling error, the estimated value used to judge whether the process capability attains the customer's requirement or not is biased. Therefore, the analytical method proposed by Vännman is used to plot the united confidence interval for $Q_{i f}$ and $Q_{i s}$ on the multi-process capability analysis chart in this paper. The proposed method uses the unified confidence interval to evaluate whether or not the indices of $Q_{i f}$ and $Q_{i s}$ meet the required values. The procedure is given in detail in the following.
Assuming that there are $k$ types of steel that can be produced by any one of the steelworks, it shows that there are $k$ types of production process in the steelworks. The appropriate tensile strength value $T$ for $k$ types of steel, the tolerance error value $d$ and the statistical values for sampling are given in Table 4 .

$$
\bar{X}_{i}=\left\{\sum_{j=1}^{n} X_{i j} / n_{i}\right\} \text { and } s_{i}=\left\{\sum_{j=1}^{n}\left(X_{i j}-\bar{X}_{i}\right) /\left(n_{i}-1\right)\right\}^{\frac{1}{2}}
$$

are used to estimate population mean $\mu_{i}$ and population standard deviation $\sigma_{i}$, respectively. Then, unbiased estimators of $Q_{i f}$ and $Q_{i s}$ are:

$$
\begin{aligned}
& \hat{Q}_{i f}=\frac{\bar{X}-T}{d} \\
& \hat{Q}_{i s}=\frac{S}{d c_{4}}
\end{aligned}
$$

where $c_{4}=\sqrt{(2 /(\mathrm{n}-1)} \Gamma[\mathrm{n} / 2] / \Gamma[(\mathrm{n}-1) / 2]$ is a function of sample size $n_{i}$, since the sample size $n_{i}$ is large enough, $c_{4}$ will approach $1 . \hat{Q}_{i f}$ is distributed as a normal distribution with a mean of $Q_{i f}$ and a variance of $\left(Q_{i s}{ }^{2}\right) / n$ based on the assumption of normality and is given by $\hat{Q}_{i f} \sim N\left(Q_{i f},\left(Q_{i s}\right)^{2} / n\right)$. The statistics $(n-1)\left\{\left[\mathrm{c}_{4} \hat{Q}_{i s}\right] / Q_{i s}\right\}^{2}$ is distributed as a chi-squared distribution with an $n-1$ degree of freedom. According to the distribution of $\hat{Q}_{i f}$ and $\hat{Q}_{i s}$, the united confidence interval of $Q_{i f}$ and $Q_{i s}$ can be expressed as follows:

$$
\begin{array}{r}
Q_{i f}:\left[\hat{Q}_{i f}-t_{\alpha / 4}(n-1) \times c_{4} \times \frac{\hat{Q}_{i s}}{\sqrt{n}},\right. \\
\left.\hat{Q}_{i f}+t_{\alpha / 4}(n-1) \times c_{4} \times \frac{\hat{Q}_{i s}}{\sqrt{n}}\right]
\end{array}
$$

$Q_{i s}:\left[\sqrt{\frac{(n-1) \times c_{4}^{2} \times \hat{Q}_{i s}^{2}}{\chi_{\alpha / 4}^{2}(n-1)}}, \sqrt{\frac{(n-1) \times c_{4}^{2} \times \hat{Q}_{i s}^{2}}{\chi_{1}^{2}-\alpha / 4}(n-1)}\right]$

where,

$t_{\alpha / 4}(n-1)$ is the upper $\alpha / 4$ percentile of the $t$ distribution with an $n-1$ degree of freedom,

$\chi_{1-\alpha / 4}^{2}(n-1)$ is the upper $1-\alpha / 4$ percentile of the chisquared distribution with an $n-1$ degree of freedom,

$\chi_{\alpha / 4}^{2}(n-1)$ is the upper $\alpha / 4$ percentile of the chi-squared distribution with an $n-1$ degree of freedom.

The united confidence interval can be established based on the requirements of the customer, as in Table 3. For instance, when the required specification of steel is $1.33 \leq S_{p k}<1.50$ (satisfactory), the contour lines of $S_{p k}=1.33$ and $S_{p k}=1.50$ can be plotted on a multi-process capability analysis chart, as shown in Fig. 1. There are five production processes shown in Fig. 1. The analyses of these five process capabilities are discussed as follows:

1. The united confidence interval for production process $a$ is totally located in block I. The process capability is inadequate. If the coordinate of confidence interval is situated in the middle, it indicates that the average tensile strength of the steel is appropriate. If the coordinate of confidence interval is inclined toward a high value, it shows that the 
Table 4. The statistics values of sampling from the tensile strength of $k$ types of steel.

\begin{tabular}{|c|c|c|c|c|c|c|c|}
\hline 1 & $T_{1}$ & $d_{1}$ & $X_{11}, \ldots$ & $X_{1 j}, \ldots$ & $X_{1 n_{1}}$ & $\bar{X}_{1}$ & $s_{1}$ \\
\hline$\vdots$ & $\vdots$ & $\vdots$ & $\vdots$ & $\vdots$ & $\vdots$ & $\vdots$ & $\vdots$ \\
\hline$\vdots$ & $\vdots$ & $\vdots$ & $\vdots$ & $\vdots$ & $\vdots^{m_{i}}$ & $\vdots$ & $\vdots$ \\
\hline$m$ & $T_{m}$ & $d_{m}$ & $X_{m 1}, \ldots$ & $X_{m j}, \ldots$ & $X_{m n_{m}}$ & $\bar{X}_{m}$ & $s_{m}$ \\
\hline
\end{tabular}

average tensile strength of steel is unstable. Engineers should find the reasons and improve it.

2. The united confidence interval for production process $b$ is situated in block C. Although the process capability is not too low, the process capability does not meet the quality control requirement. If the position of the coordinate of confidence interval inclines toward the left, then it indicates that the average tensile strength of steel is close to a low value, which is unsatisfactory. Engineers should investigate the cause and improve it.

3. The united confidence interval for production process $c$ is located on the contour line of $S_{p k}=1.33$. The process capability attains the requirement. Although the process capability meets the requirement, the engineer should especially supervise changes in this process.

4. The united confidence interval for production process $d$ is totally located in block $\mathrm{S}$. The process capability meets the requirement.

5. The united confidence interval for production process $e$ is totally situated in block $U$. The process capability not only meets the requirement, but also exceeds the requirement. The steelworks can now consider a lower cost process to replace the current production process, or review and discuss the specification.

According to the five analyses of process capability, the location of the united confidence interval for process and the corresponding indications are arranged in Table 5.

\section{The Investigation of Examples}

The fitness or unfitness of steel quality greatly affects the safety of reinforced concrete structures. In order to ensure that the steel reinforcing bars contribute suitable tensile strength and ductility for the reinforced concrete structure, the ACI code has prescribed a yield strength range for the steel bars. To increase profit and lower the rate of rejection of inferior goods, the deviation of a product is lowered to pass quality control inspection. The evaluation method proposed in this paper considers the quality control and process capability of steelworks. This evaluation method can control the suitability of a product. To show this evaluation method clearly, an example on the test tensile strength of steel is discussed below. These reports are based on the mechanical properties from a mill report. Each numerical test represents the mechanical property of steel in each stove. SD42 is used for the main bars; the numbers of the steel used in this test data are D22 and D32; and the design yield stress is $42 \mathrm{kgf} \mathrm{mm}^{-2}$. SD28 is used for ties and the steel used in this test data is D10 and D16, and the design yield stress is $28 \mathrm{kgf} \mathrm{mm}^{-2}$. In order to avoid too much difference between the actual yield stress and the design yield stress, the Building Code Requirements for Reinforced Concrete and Commentary [15], section 21.2.5, "Reinforcement in members resisting earthquake-induced forces" specifies that the actual yield strength of ASTM A 615 Grades 40 and 60 reinforcement should not exceed the specified yield strength by more than $12.6 \mathrm{kgf} \mathrm{mm}^{-2}$. Under the provision of ACI building code 21.2.5, the target value $T$, the tolerance error $d$, the upper tolerance limit $U T L$ and the lowest tolerance limit $L T L$ are given in Table 6.

The following data of the mill report for tensile strength of steel are used to evaluate the production and process capability of the steelworks. There are 120 samples of test tensile strength for each steel number. The mean values, variance values of samples, and the unbiased estimator $\hat{Q}_{i f}, \hat{Q}_{i s}$ and the value of. United confidence interval for $Q_{i}, Q_{i s}$ are calculated by the above-mentioned equations (Table 7).

The results are plotted in the multi-process capability chart shown in Fig. 2, and the conclusions are discussed below:

Table 5. The location of united confidence interval for process and the correspondence of stratagem.

\begin{tabular}{|c|c|c|c|}
\hline I & Inadequate & No $\square$ & $\begin{array}{l}\text { Need urgently to } \\
\text { improve }\end{array}$ \\
\hline $\mathrm{C}$ & Capability & Yes $\square$ /No $\square$ & Maintain/improve \\
\hline S & Satisfactory & Yes $\square$ /No $\square$ & Maintain/improve \\
\hline $\mathrm{E}$ & Excellent & Yes $\square$ /No $\square$ & Maintain/improve \\
\hline
\end{tabular}


Table 6. The specification of steel reinforcing bars.

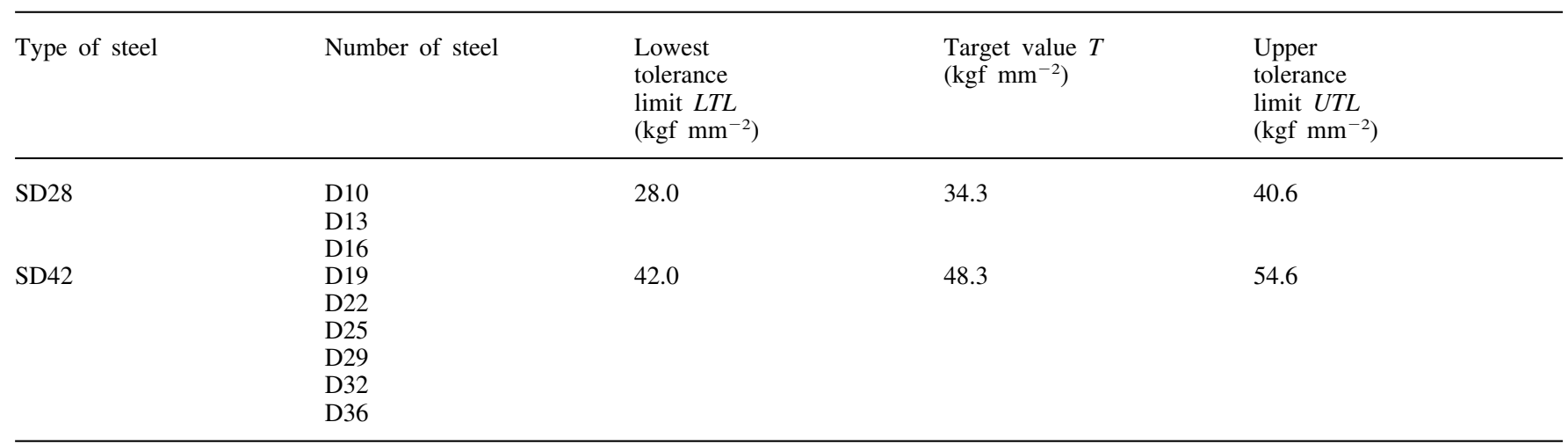

Table 7. The mill report of tensile strength of each type of steel, unbiased estimator and united confidence interval.

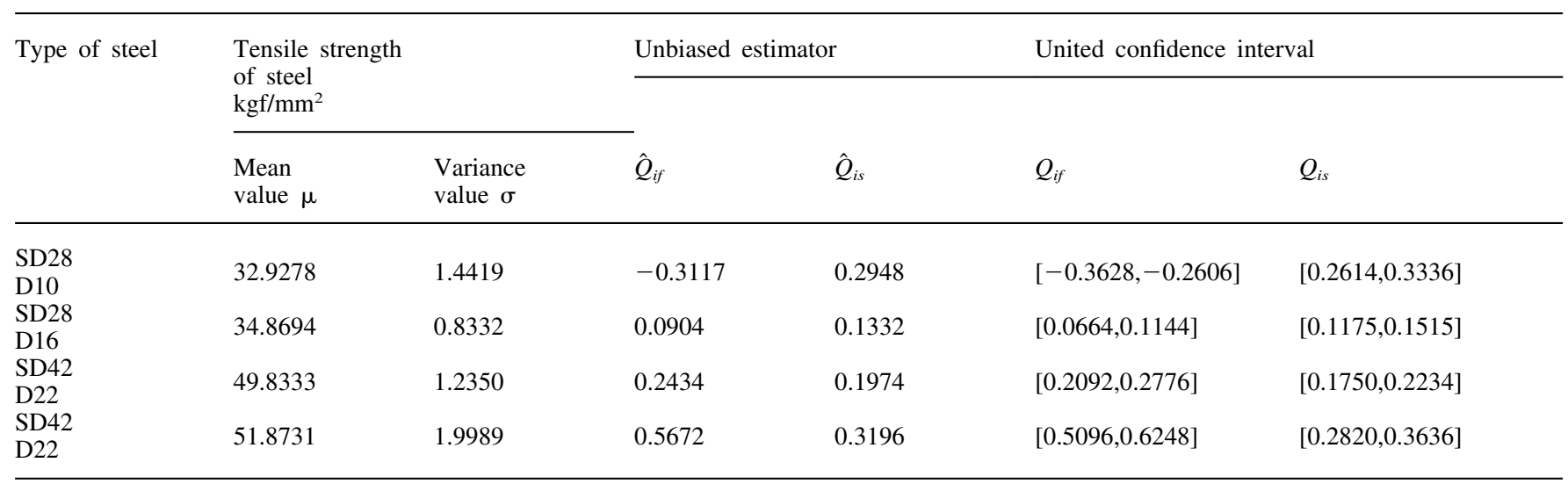

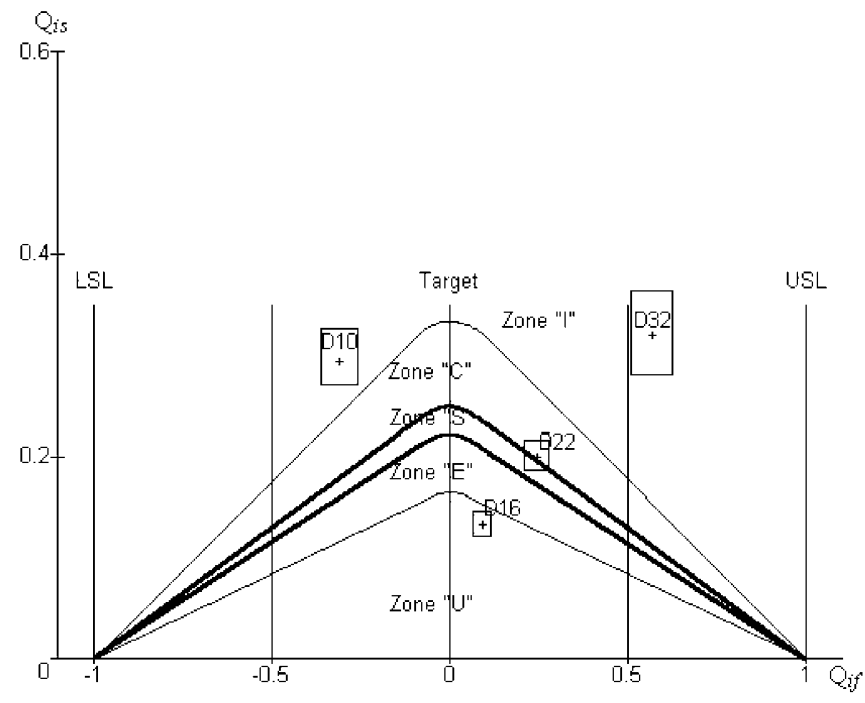

Fig. 2. The analysis results of a mill report of tensile strength of stee plotted in a multi-process capability analysis chart.
1. The block of united confidence interval for SD 28 D10 is located near the contour line of $S_{p k}=1.00$. This process capability approaches the requirement. In order to prevent unusual conditions happening in this process, the engineer should strictly supervise this process and follow previous examples.

2. The block of united confidence interval for SD 28 D16 is situated on the $U$ block. It indicates that this process is an excellent process and the average value approaches the target value. It exceeds the qualified process requirement. Under the primary consideration of permitting no influence on total quality, a new plan for a lower cost material and process are sought. The purpose of this new plan is to attain the requirement of appropriate quality of the product and also to reduce cost and thus increase the profitability of the product.

3. The block of united confidence interval for SD 42 D22 is totally situated on the $\mathrm{S}$ block. The process capability attains the requirement and suggests that the production process should be maintained.

4. The block of united confidence interval for SD 42 D22 is located on the I block. It reveals that this process capability 
is inadequate. To understand the deviation of this process and put forward a proposal, the target value should be used as a goal and this process should be re-examined and follow the examples of predecessors.

\section{Conclusions}

Good or bad quality of steel greatly affects the safety of structures. The engineering unit now tests the steels by random selection. To ensure that the steel provides the appropriate tensile strength and ductility for the structure, the ACI code prescribes a yield strength range for steel bars. The safety of a structure is affected by discrepancies in tensile strength and quality. This research continues the work of Sung et al. [11] and develops a new evaluation method. This method plots the indices of the various steel types on a multi-process capability chart. The production level, deviation degree, and the influence of various types of steel can be evaluated easily by the indices and improvements in the process capability can be proposed.

The multi-process capability analysis chart can not only be used for the specification of the various production processes, but it also conveys the rate of tallied tolerance relationship between production processes and each of the quality characteristics. This method provides equations to obtain the production process for each type of steel. In addition, it offers a set of procedures for the construction industry and steelworks to evaluate the steel quality easily. This evaluation method helps the construction industries to make purchase decisions. It also offers the steelworks an analytical method for improving production process and quality control capability. This analysis method is a convenient and effective tool.

\section{Acknowledgement}

The National Science Council, Taiwan, supported this research through grant no. NSC 89-2213-E-167-004. This support is gratefully acknowledged.

\section{References}

1. M. Watabe, "Lessons Learnt from Hanshin-Awaji Devastating Earthquake," Conference of Architecture Damage on HanshinAwaji Earthquake, Taipei, Taiwan, pp. 1-1-1-49, 1995.

2. National Center for Research on Earthquake Engineering, Taiwan, The Investigation of 921 Chi-Chi Earthquake Damages, NCREE, Taiwan, 1999.

3. V. E. Kane, "Process capability indices," Journal of Quality Technology, 18(1), pp. 41-52. 1986.

4. L. K. Chan, S. W. Cheng and F. A. Spiring, "A new measure of process capability: $C_{p m}$ ", Journal of Quality Technology, 20, pp. $162-175,1988$

5. Y. M. Chou and D. B. Owen, "On the distribution of the estimated process capability indices," Communications in Statistics Theory and Methods, 18, pp. 4549-4560, 1989.

6. R. A. Boyles, "The Taguchi capability index," Journal of Quality Technology, 23(1), pp. 17-26, 1991.

7. W. L. Pearn, S. Kotz and K. L. Johnson, "Distribution and inferential properties of process capability indices," Journal of Quality Technology, 24, pp. 216-231, 1992.

8. R. A. Boyles, "Process capability with asymmetric tolerances," Journal of Tolerances, Communications in Statistics Simulation and Computation, 23(3), pp. 615-643, 1994.

9. M. Greenwich and B. L. Jahr-Schaffrath, "A process incapability index," International Journal of Quality and Reliability Management, 12(4), pp. 58-71, 1995.

10. K. S. Chen, "Incapability index with asymmetric tolerances," Statistica Sinica, 8, pp. 253-262, 1998.

11. W. P. Sung, K. S. Chen and C. C. Go, "The effective method of evaluation for the quality control of steel reinforcing bar," Advances and Applications in Statistics, 1(2), pp. 175-193, 2001.

12. K. Vännman and M. Deleryd, "Process capability plots - A quality improvement tool," Quality and Reliability International, 15, pp. 213-217, 1999.

13. W. L. Pearn and K. S. Chen, "Multiprocess performance analysis: a case study," Quality Engineering, 10(1), pp. 1-8, 1998.

14. P. L. Chang, S. N. Hwang and W. Y. Cheng, "Using data envelopment analysis to measure the achievement and change of regional development in Taiwan," Journal of Environmental Management, 43, pp. 49-66, 1995.

15. ACI committee 318, Building Code Requirement for Reinforced Concrete (ACI 318-95) and Commentary - ACI 318R-95, ACI Committee 318, 1996 